\title{
BMJ Open Management of asthma in childhood: study protocol of a systematic evidence update by the Paediatric Asthma in Real Life (PeARL) Think Tank
}

Alexander G Mathioudakis (1) , ${ }^{1,2,3}$ Michael Miligkos, ${ }^{4}$ Cristina Boccabella, ${ }^{5}$ Gioulinta S Alimani, ${ }^{3,6}$ Adnan Custovic, ${ }^{7}$ A Deschildre, ${ }^{8}$ Francine Monique Ducharme, ${ }^{9}$ Omer Kalayci, ${ }^{10}$ Clare Murray, ${ }^{1,2}$ Antonio Nieto Garcia, ${ }^{11}$ Wanda Phipatanakul, ${ }^{12}$ David Price, ${ }^{13,14}$ Aziz Sheikh, ${ }^{15}$ loana Octavia Agache, ${ }^{16}$ Leonard Bacharier, ${ }^{17}$ Apostolos Beloukas (D) ,,18 Andrew Bentley, ${ }^{2,19}$ Matteo Bonini,, ${ }^{5,20}$ Jose A Castro-Rodriguez, ${ }^{21}$ Giuseppe De Carlo, ${ }^{22}$ Timothy Craig, ${ }^{23}$ Zuzana Diamant, ${ }^{24,25,26}$ Wojciech Feleszko, ${ }^{27}$ Tim Felton, ${ }^{1,2}$ James E Gern, ${ }^{28}$ Jonathan Grigg, ${ }^{29}$ Gunilla Hedlin, ${ }^{30}$ Elham M Hossny, ${ }^{31}$ Despo lerodiakonou, ${ }^{32}$ Tuomas Jartti, ${ }^{33}$ Alan Kaplan, ${ }^{34}$ Robert F Lemanske, ${ }^{28}$ Peter N Le Souëf, ${ }^{35}$ Mika J Mäkelä, ${ }^{36}$ Georgios A Mathioudakis, ${ }^{3}$ Paolo Matricardi, ${ }^{37}$ Marina Mitrogiorgou, ${ }^{38}$ Mario Morais-Almeida, ${ }^{39}$ Karthik Nagaraju, ${ }^{40}$ Effie Papageorgiou, ${ }^{6}$ Helena Pité, ${ }^{39,41,42}$ Paulo M C Pitrez, ${ }^{43}$ Petr Pohunek, ${ }^{44}$ Graham Roberts, ${ }^{45,46,47}$

To cite: Mathioudakis AG, Miligkos M, Boccabella C, et al. Management of asthma in childhood: study protocol of a systematic evidence update by the Paediatric Asthma in Real Life (PeARL) Think Tank. BMJ Open 2021;11:e048338. doi:10.1136/ bmjopen-2020-048338

- Prepublication history and additional online supplemental material for this paper are available online. To view these files, please visit the journal online (http://dx.doi.org/10. 1136/bmjopen-2020-048338).

AGM and MM contributed equally.

Received 23 December 2020 Accepted 01 June 2021

\section{Check for updates}

(c) Author(s) (or their employer(s)) 2021. Re-use permitted under CC BY-NC. No commercial re-use. See rights and permissions. Published by BMJ.

For numbered affiliations see end of article.

\section{Correspondence to} Professor Nikolaos G Papadopoulos; ngpallergy@gmail.com

loanna Tsiligianni, ${ }^{32}$ Stephen Turner, ${ }^{48}$ Susanne Vijverberg, ${ }^{49}$ Tonya A Winders, ${ }^{50}$ Gary WK Wong (D) , ${ }^{51}$ Paraskevi Xepapadaki, ${ }^{52}$ Heather J Zar, ${ }^{53,54}$ Nikolaos G Papadopoulos (i) ${ }^{1,52}$

\section{ABSTRACT}

Introduction Clinical recommendations for childhood asthma are often based on data extrapolated from studies conducted in adults, despite significant differences in mechanisms and response to treatments. The Paediatric Asthma in Real Life (PeARL) Think Tank aspires to develop recommendations based on the best available evidence from studies in children. An overview of systematic reviews (SRs) on paediatric asthma maintenance management and an SR of treatments for acute asthma attacks in children, requiring an emergency presentation with/without hospital admission will be conducted.

Methods and analysis Standard methodology recommended by Cochrane will be followed. Maintenance pharmacotherapy of childhood asthma will be evaluated in an overview of SRs published after 2005 and including clinical trials or real-life studies. For evaluating pharmacotherapy of acute asthma attacks leading to an emergency presentation with/without hospital admission, we opted to conduct de novo synthesis in the absence of adequate up-to-date published SRs. For the SR of acute asthma pharmacotherapy, we will consider eligible SRs, clinical trials or real-life studies without time restrictions. Our evidence updates will be based on broad searches of Pubmed/Medline and the Cochrane Library. We will use A MeaSurement Tool to Assess systematic Reviews, V.2, Cochrane risk of bias 2 and REal Life EVidence AssessmeNt Tool to evaluate the methodological quality

\section{Strengths and limitations of this study}

Broad evidence syntheses on the management of childhood asthma, with a focus on the differential treatment response according to age and disease phenotypes could reveal clinically exploitable information, that will be used in the development of clinical and research recommendations by Paediatric Asthma in Real Life.

- A rigorous methodology that includes thorough evaluation of the literature, appropriate evaluation of the methodological quality of individual studies andwhen appropriate- of the body of evidence, and presentation of overall effect estimates.

- A prospectively published protocol increases the transparency and allowed for peer-review of the methodology used.

- A potential limitation of the overview of systematic reviews (SRs) is that the feasibility of conducting the planned subgroup analyses will depend on whether relevant data have been captured in existing SRs.

of SRs, controlled clinical trials and real-life studies, respectively.

Next, we will further assess interventions for acute severe asthma attacks with positive clinical results in metaanalyses. We will include both controlled clinical trials and observational studies and will assess their quality 
using the previously mentioned tools. We will employ random effect models for conducting meta-analyses, and Grading of Recommendations Assessment, Development and Evaluation methodology to assess certainty in the body of evidence.

Ethics and dissemination Ethics approval is not required for SRs. Our findings will be published in peer reviewed journals and will inform clinical recommendations being developed by the PeARL Think Tank.

PROSPERO registration numbers CRD42020132990, CRD42020171624.

\section{INTRODUCTION}

Having a global prevalence that is anticipated to exceed 400 million children by the year 2025, childhood asthma represents a huge health and socioeconomic burden to patients, their families and the society. ${ }^{1-3}$ Despite its diverging mechanisms, triggers, outcomes and response to treatment, childhood asthma is often still approached as an extension of adult asthma. ${ }^{4}$ It is underaddressed in clinical guidelines, likely due to unclear diagnosis, limited availability of safety, efficacy and effectiveness data in this population. Clinical recommendations are to a large extent informed by data extrapolated from clinical studies conducted in adults. ${ }^{2-5}$

Numerous challenges complicate conducting interventional research studies in children with asthma. Besides the lack of consensus on its definition and diagnostic criteria, childhood asthma is highly heterogeneous and our understanding of different paediatric asthma phenotypes is still limited or contradictory. ${ }^{6}$ This is further emphasised by significant variability in disease progression, outcomes and treatment response in children with different phenotypes or ages $5,{ }^{7}$ potentially complicating interpretation of trials' findings. In addition, there are regulatory and ethical constraints in conducting interventional research in children. ${ }^{8}{ }^{9}$ However, this results in the administration of treatments that have not been adequately evaluated in relevant (paediatric) populations, that is, evidently suboptimal.

Paediatric Asthma in Real Life (PeARL), an international Think Tank focusing on paediatric asthma, was initiated in the context of the respiratory effectiveness group, to address this evidence deficit. In a recent international, multistakeholder survey, we have identified and prioritised unmet needs on paediatric asthma. ${ }^{10} \mathrm{~A}$ need for systematic evidence updates focusing on the management of asthma in different age groups emerged. Herein, we present the protocol for a series of systematic evidence updates aiming to summarise direct evidence from clinical studies in children with asthma, evaluating the safety and clinical effectiveness of pharmacological interventions for maintenance management and for the treatment of acute severe asthma attacks, defined as those leading to an emergency presentation with/without hospital admission, in different age groups. Our work will be used to inform clinical recommendations being developed by the PeARL Think Tank. Therefore, we need solid evidence on the efficacy on safety of various interventions. It is considered crucial to incorporate evidence derived from real-life observational studies, which may carry a lower strength of evidence than randomised controlled trials (RCTs), but are available in higher abundance and provide a better representation of clinical practice in real life, where for example, treatment compliance or inhaler technique may be problematic.

\section{METHODS AND ANALYSIS}

We will conduct two systematic evidence updates, based on protocols prospectively registered in the PROSPERO register (CRD42020132990, ${ }^{11}$ CRD42020171624 ${ }^{12}$ ). The first will evaluate the safety and clinical effectiveness of pharmacological maintenance treatments for childhood asthma, while the other will focus on the pharmacotherapy of acute severe asthma attacks, defined as those requiring a hospital admission or emergency presentation. We will use standard methodology recommended by the Cochrane Collaboration ${ }^{13}$ and will follow the Preferred Reported Items for Systematic Reviews and Meta-Analyses statement. ${ }^{14}$

Preliminary searches revealed several RCTs evaluating maintenance pharmacotherapy of childhood asthma, which have already been summarised in highquality systematic reviews (SRs), some conducted by the Cochrane Collaboration. We identified $>40$ up-to-date SRs evaluating inhaled corticosteroids (ICS), long-acting beta-2 agonists (LABA), long-acting muscarinic antagonists (LAMA), leukotriene receptor antagonists (LTRA) or biologic therapies, as first line or add-on treatment for asthma in children. As a result, we opted to produce an overview of existing SRs of clinical trials and real-life studies. ${ }^{15}$

We found less up-to-date SRs on the management of acute severe asthma attacks in children, mainly focusing on short-acting beta-2 agonists (SABA), short acting muscarinic antagonists, oral corticosteroids, aminophylline and magnesium that were recently summarised in a Cochrane Overview of SRs. ${ }^{16}$ However, when evaluating the literature, we identified several other pharmacological interventions that are tested in small trials or real-life studies, and while they may show promising early results, they have not been assessed further or introduced in clinical practice guidelines. ${ }^{17-23}$ For this reason, we will conduct de novo synthesis of comparative clinical studies of any design aiming to identify any pharmacological intervention that has been tested for acute severe asthma attacks, followed by focused meta-analyses of promising interventions not covered by existing high-quality SRs or clinical practice guidelines.

Overview of SRs evaluating maintenance pharmacotherapy for paediatric asthma

Eligibility criteria

Eligible studies will comprise SRs and meta-analyses of controlled clinical trials or of real-life studies evaluating maintenance treatments that are broadly used in clinical practice for asthma or recurrent wheeze in children 
and adolescents, aged up to 18 years. More specifically, we will include SRs comparing any combination of ICS, LABA, LAMA, LTRA, biological therapies (namely omalizumab, mepolizumab, reslizumab, benralizumab or dupilumab), or placebo as monotherapy or add-on maintenance therapy for paediatric asthma. We will accept SRs and meta-analyses evaluating any molecule of the abovementioned categories, administered at any dose and for a duration of at least 6 weeks. SRs comparing asthma maintenance treatment both in children and adults will be included provided that paediatric data are presented separately. We will only include SRs published between 2005 and December 2020 and reported in the English language. Older SRs are probably outdated and will only be considered in the absence of high-quality, newer SRs.

\section{Outcome measures}

The primary outcomes of this overview will be the number of acute attacks requiring the administration of oral corticosteroids or an emergency visit, and the number of acute attacks requiring hospitalisation. Secondary outcomes will include lung function measures, acute attacks irrespective of the severity, symptom scores (including symptom free and rescue medication free days), asthma control, asthma-specific quality of life scores, use of rescue medications, withdrawal rates (overall, due to lack of efficacy or adverse events), adverse events and serious adverse events.

\section{Search strategy and study selection}

The electronic databases of Medline/PubMed and Cochrane Library will be systematically searched, using appropriate controlled vocabulary and free search terms to identify relevant SRs (terms describing: childhood asthma, LABA, LAMA, LTRA, ICS, biologics, SRs, detailed search strategy is available in online supplemental appendix). Databases will be searched from 2006 onwards. Titles and abstracts of all identified manuscripts, and the full texts of potentially relevant manuscripts, will be screened by two investigators independently. We will report the reasons of exclusion of studies that will be excluded after full-text review. Disagreement will be resolved through discussion or adjudication by a third investigator, when necessary.

\section{Data abstraction}

For each of the included SRs, one investigator will extract the full reference and study identifiers, references of the included trials evaluating paediatric populations, eligibility criteria, predefined outcomes, number and baseline characteristics of the participants and details on the outcomes of interest. A second investigator will crosscheck for validity.

\section{Risk of bias assessment}

A MeaSurement Tool to Assess systematic Reviews, V.2 (AMSTAR 2) tool will be used to evaluate the methodological quality of all included SRs. ${ }^{24}{ }^{25}$ The AMSTAR 2 tool evaluates 16 domains, focusing on the methodological design, interpretation and potential risk of bias involved in the conduct of a SR. It is considered by the AMSTAR 2 team that seven domains could critically affect the validity of the review, while the remaining domains describe noncritical weaknesses. Critical flaws for an SR include (1) lack of prospective protocol registration, (2) inadequate literature searches, (3) lack of justification of excluding individual studies, (4) of risk of bias evaluation or (5) of risk of bias consideration in interpreting the results, (6) of assessment of presence and likely impact of publication bias and (7) inadequate methodology for conducting meta-analysis. We will consider the results of an SR of high quality, if there is only one or none non-critical weakness, and of moderate quality, if there are more than one noncritical weaknesses. If there are one or more critical weaknesses, then we will consider the confidence low or very low, respectively. Two of the SRs will evaluate the risk of bias independently and disagreement will be resolved through discussion, or adjudication by a third reviewer.

\section{Qualitative synthesis}

We will summarise descriptively or in a tabulated format the characteristics of the included SRs and outcomes of interest. When several SRs evaluate the same intervention, we will compare their eligibility criteria, included studies and methodological quality as evaluated by the AMSTAR-2 tool, as well as the pertinent subgroup analyses that are presented. We will present in detail the results of the SR that is most recent, more complete and of high methodological quality. If no single SR fulfil these criteria, we will present in detail more than one SRs. From the remaining SRs, we will present pertinent additional information that may include, such as details about additional outcomes, or additional subgroups.

We will specifically report on the differential effectiveness of the interventions across different maintenance treatment steps (severity), age groups or paediatric asthma phenotypes.

\section{SR of clinical studies evaluating the management of acute severe asthma attacks}

Over the past decades, several interventions have been tested for the management of acute severe asthma attacks, such as ketamine or macrolide antibiotics. ${ }^{17-23}$ Despite promising early findings, some of these interventions were not further tested in robust, prospective controlled clinical trials. This may partially be due to challenges in conducting experimental clinical studies in children, as previously discussed, particularly during acute, lifethreatening conditions.

To identify all evaluated treatments, a two-stage approach will be followed. First, a broad search strategy will be used to identify all pharmacological interventions that have been tested as potential treatments for acute severe asthma attacks. Next, medications that showed positive clinical results, but are not yet thoroughly evaluated in clinical studies and meta-analyses and are therefore not recommended by international asthma 
guidelines (such as the National Institute for Health and Care Excellence asthma guidelines, the British Thoracic Society and Scottish Intercollegiate Guidelines Network asthma guidelines, the National Asthma Education and Prevention Programme or the Global Strategy for Asthma Management and Prevention document), will be selected and further evaluated in individual meta-analyses. The aim will be to identify novel interventions that could be recommended for use in clinical practice, or might require further evaluation in clinical research studies, to confirm their safety and effectiveness profiles.

\section{BROAD SR}

Medline/PubMed and the Cochrane Library will be searched, using a broad search strategy, aimed to identify any clinical research studies evaluating the management of acute severe asthma attacks (detailed search strategy is available in online supplemental appendix).

Any study evaluating pharmacological treatments for acute severe asthma attacks in children and adolescents $(<18$ years of age) will be included. Any comparative clinical research study, including experimental and observational studies, as well as SRs of such studies will be considered eligible for inclusion. We will only include studies published until May 2021 and reported in the English language, without time restrictions.

Eligible studies will be grouped according to the drug category they evaluate and will be presented narratively. Study design, characteristics and outcomes of interest will be reported descriptively or in a tabulated format. Outcomes of interest are the same for this broad SR and individual medication meta-analyses and are detailed in the next section.

\section{INDIVIDUAL MEDICATION META-ANALYSES}

These meta-analyses will further evaluate the safety and clinical effectiveness of individual medications that were assessed by the initial broad SR and were found to be of potential clinical value for the treatment of acute severe asthma attacks. In contrast to most preceding SRs and meta-analyses, we will include data from observational comparative effectiveness (real-life) studies, as well as controlled clinical trials.

\section{Eligibility criteria}

For each meta-analysis, eligible studies will comprise controlled clinical trials and observational comparative effectiveness studies comparing the index medication with placebo, no treatment or any active control, as an add-on treatment for acute severe asthma attacks. Index medication will be defined based on the pharmacological action, meaning that molecules targeting the same pharmacological target (eg, salbutamol and terbutaline, both being SABA) will be grouped. Only studies evaluating the management of acute severe asthma attacks, defined as those requiring a hospital admission or emergency presentation, in children and adolescents, aged between 1 and 18 years of age will be included. Studies evaluating both children and adults will be included, provided that paediatric data are reported separately or that we will be able to access these data after requesting them from the investigators. We will only include observational studies that meet the primary criteria of the REal Life EVidence AssessmeNt Tool (RELEVANT) tool (see risk of bias). We will include studies published until May 2021 and reported in the English language.

\section{Outcome measures}

The primary outcome measures will be (1) treatment success or treatment failure rate evaluated at any time point, within 2 weeks from presentation, (2) serious adverse events and (3) need for asthma related hospitalisation evaluated at any tim epoint within 2 weeks from presentation. Treatment success will be defined as a complete resolution of the symptoms, or an improvement in the clinical signs, symptoms and/or laboratory findings that fulfils specific criteria or thresholds prespecified by the study team. Treatment failure will be defined as a significant deterioration of the patients' clinical conditions that fulfils specific criteria prespecified by the study team. For example, treatment failure may be defined as the need for paediatric intensive care unit admission, ventilation or death. The definitions of treatment success and treatment failure vary significantly across clinical studies evaluating the management of acute asthma in children; for this reason, meta-analyses will only be conducted in cases they are considered meaningful by the investigators. Need for asthma-related hospitalisation will not be relevant for studies only evaluating hospitalised participants. Secondary outcomes will include (1) mortality, (2) duration of asthma-related hospitalisation, (3) need for intensive care unit admission, (4) duration of intensive care unit stay, (5) re-exacerbation rate, (6) rehospitalisation rate and (7) adverse events. All outcomes will be evaluated at a maximum follow-up of 6 months, as longerterm outcomes are less likely to be directly linked with the index acute event.

\section{Search strategy and study selection}

Using appropriate controlled vocabulary and free search terms, we will systematically search Medline/PubMed, EMBASE and the Cochrane Library to identify controlled clinical trials and observational comparative effectiveness studies evaluating the safety, efficacy and/or clinical effectiveness of the selected medication (sample search strategies are available in the online appendix). We will also search the WHO International Clinical Trials Registry Platform search portal, the abstract proceedings of the European Respiratory Society, the American Thoracic Society, the Asian Pacific Society of Respirology, the European Academy of Allergy and Clinical Immunology, the American Academy of Allergy, Asthma and Immunology, and the World Allergy Organization, as well as the reference lists of all included studies. All sources 
will be searched from inception, without language limitations. We will follow standard methodology for screening titles, abstracts and the full text of all identified studies, as described previously.

\section{Data abstraction}

The full study reference, study identifiers, details on the study design, eligibility criteria, predefined outcomes and potential confounding factors that were considered by the investigators, number and baseline characteristics of participants will be extracted by one investigator and will be cross-checked for validity by a second extractor. Details on the outcomes of interest from all included studies will be extracted by two investigators independently. Conflicts will be resolved through discussion and when needed adjudication by a third investigator.

\section{Risk of bias of individual studies}

We will use the second version of the Cochrane risk of bias (RoB2) tool for assessing risk of bias in the included RCTs $^{26}$ and the RELEVANT for assessing the risk of bias of observational studies. ${ }^{27}$ Risk of bias of each included study will be evaluated by two investigators independently.

The RoB2 tool evaluates the following domains for potential risk of bias: (1) bias arising from the randomisation process, (2) bias due to deviations from intended interventions, (3) bias due to missing outcome data, (4) bias in measurement of the outcome, (5) bias in selection of reported results and (6) any other potential source of bias. High risk of bias in any of these domains will result in an overall judgement of high risk of bias. In the absence of high-risk domains, unclear risk in any domain will lead to an overall judgement of unclear risk. All remaining trials will be considered to be of low risk of bias.

RELEVANT evaluates the quality of observational comparative effectiveness research studies across seven domains, which include background, design, measures, analysis, results, discussion/interpretation and conflicts of interest. Each domain includes primary and secondary items. It is suggested that studies not meeting the primary items of RELEVANT are of very low methodological quality (have 'fatal flaws') and should not be used to inform clinical recommendations. Therefore, we will exclude studies not meeting these criteria. We will consider of low risk of bias all studies meeting the secondary criteria of RELEVANT as well, and of high risk of bias studies that do not meet any of the secondary criteria.

For every comparison, we will use funnel plots, Egger's regression and Begg's rank tests to evaluate publication bias, if we are able to pool more than 10 studies.

\section{Data synthesis}

Data from controlled clinical trials or observational studies will be analysed separately. In addition, studies evaluating different comparators, will be analysed separately. If different doses of the index medication or comparator are evaluated across the included studies, we will consider grouping studies using similar doses, providing that their results are not significantly dissimilar.

For every analysis, $\mathrm{I}^{2}$ statistic will be used to assess statistical heterogeneity. Substantial heterogeneity $\left(\mathrm{I}^{2}>50 \%\right)$ will be explored using prespecified subgroup analyses (details in the next section). We will not perform metaanalyses in cases of considerable unresolved heterogeneity $\left(\mathrm{I}^{2}>75 \%\right)$.

When it is considered meaningful, meta-analyses will be performed using the random-effects model, because we anticipate significant heterogeneity in our data. Results will be presented in the form of relative risk (95\% CI) for dichotomous data, mean difference $(95 \% \mathrm{CI})$ for continuous data and (HR, 95\% CI) for time to event data. Meta-analyses will be performed using Review Manager V.5 (RevMan, http://community.cochrane.org/tools/ review-production-tools/revman-5) and $\mathrm{R}$ statistics V.3.4.3 or newer (R Foundation for Statistical Computing, Vienna, Austria).

For dichotomous outcomes, the unit of analysis will preferably be participants, rather than events (ie, number of participants admitted to the intensive care unit, rather than number of admissions per participants).

\section{Sensitivity and subgroup analyses}

In sensitivity analyses for all comparisons, we will (1) use fixed effects models, (2) only include studies with low risk of bias, (3) exclude studies reporting limited adherence to the study drugs $(<80 \%)$ and (4) evaluate separately studies assessing different doses of the index medication, which we may pool in the main analysis.

Subgroup analyses according to participants' age, asthma phenotypes or, possibly, acute attack phenotypes will also by conducted, depending on data availability. In an additional subgroup analysis, we will evaluate separately trials utilising exploratory versus pragmatic study designs.

\section{Certainty of the body of evidence}

Certainty of the body of evidence, for every comparison will be evaluated using the Grading of Recommendations Assessment, Development and Evaluation (GRADE) methodology. ${ }^{28}$ GRADE assesses the certainty in a body of evidence as high, moderate, low or very low after considering the methodological quality of the included studies, imprecision, inconsistency, indirectness, publication bias, the magnitude of effect, dose response and confounders likely to minimise the effect. All decisions to upgrade or downgrade the quality of evidence will be transparent and justified in evidence profile and summary of evidence tables, in accordance with GRADE guidance. GRADEPro Software (2014; www.gradepro.org) will be used for the development of these tables.

We will use GRADE methodology to assess the risk of bias associated with missing participant outcome data across the body of the available evidence. ${ }^{29}$ GRADE suggests repeating the primary meta-analysis, imputing the most extreme assumptions about the values of the 
missing data, that the investigators consider plausible. Only if the analyses prove robust to this imputation, the risk of bias due to missing participant outcome data should be deemed low.

The impact that the risk of bias of individual studies and the confidence in the body of the evidence has on the results will be presented.

\section{ETHICS AND DISSEMINATION}

Ethical approval is not required for these SRs, since no primary data will be collected.

The findings of these evidence updates will be presented in national and international scientific conferences. They will also be submitted for publication in high-impact peer review journals. Plain English summaries of the final reports will be developed and shared with relevant patient organisations. Moreover, our results will be used to inform clinical recommendations that will be developed by the PeARL Think Tank. We anticipate that the overview of SRs will be completed by the end of 2021 and the remaining SRs by June 2022.

\section{PATIENT AND PUBLIC INVOLVEMENT}

The planned SRs were prioritised through a global, multi-stakeholder survey evaluating research priorities in childhood asthma, conducted by the PeARL Think Tank. ${ }^{10}$ Among other stakeholders, this survey included responses from patients, patient caregivers and patient organisations. Moreover, two patient representatives (GDC and TAW) have joined the research group and provided input in this study protocol and they will also provide input throughout the study process.

\section{DISCUSSION}

We report on the methodology of a series of planned systematic evidence updates, aiming to evaluate maintenance management of childhood asthma, and the treatment of acute severe asthma attacks. Their design is informed by preliminary searches and the anticipated data availability. These SRs will be conducted by the PeARL group and will be used to inform clinical recommendations and future research needs. The need for high-quality evidence updates and clinical practice guidelines to improve the management of asthma in children is more urgent now, given the pressure that the unfolding COVID-19 pandemic pose on the healthcare systems, forcing us to reconsider our daily clinical practice. ${ }^{30} 31$

Major strengths of our evidence update series are the inclusion of a wide evidence base, including data from RCTs and real-life comparative studies, the prospective design and strong methodology. The methodological quality of all available studies will be scrutinised and will aid the interpretation of our findings. Moreover, we will attempt to evaluate differential therapeutic response of different asthma phenotypes and age groups. We believe this analysis will be revealing, if adequate data is available, but may nevertheless reveal important gaps.

Guided by the available evidence, we will follow different strategies for the evidence updates on maintenance treatment of paediatric asthma and on management of acute severe asthma attacks. In view of the availability of ample published, up-to-date SRs on maintenance pharmacotherapy of childhood asthma, we chose to conduct an overview of SRs. We decided to focus on the most frequently used and thoroughly evaluated drug classes (ICS, LABA, LAMA, LTRA and biological therapies) and we expect to identify good quality data, which would inform clinical practice and research needs. Other, less frequently or experimentally used treatments will need to be evaluated in future studies. A potential limitation of this approach is that we might not be able to capture adequate data regarding the differential effectiveness of interventions across different severity groups, age groups or paediatric asthma phenotypes, if these have not been captured in existing SRs. Moreover, existing SRs may not capture some of the most recent studies, that may have been published after the SRs, although preliminary searches have revealed several very recently update meta-analyses.

The second SR, focusing on the management of acute attacks, will first evaluate a multitude of established and experimental treatments. With regard to the latter, this SR will reveal treatments that have been tested, appeared safe and efficacious and it may be worth to be further evaluated, but will also report on interventions that were tested, but did not appear efficacious, and therefore, further evaluation may not be beneficial. This wide approach would aid the prioritisation of interventions to be further validated in future clinical research studies.

Next, meta-analyses of individual pharmacological interventions will be conducted to further assess the safety and clinical effectiveness of treatments for acute severe asthma attacks that will appear efficacious in our broad SR. In contrast to most previous meta-analyses, that may have been conducted, we will include both controlled clinical trials and observational comparative effectiveness studies. Due to limitations that have already been discussed, few controlled clinical trials are conducted in children. This leads several Cochrane SRs to report low or very low confidence in the body of evidence, due to the lack of data. ${ }^{32-35}$ We believe that by incorporating data from observational studies we may be able to conclude more robust results. While observational studies are at a higher risk of bias, we will carefully evaluate this risk using the newly developed, thorough RELEVANT tool and we will discuss potential implications on our findings. The GRADE working groups provides transparent guidance for assessing the certainty in a body of evidence including data from different study designs (controlled clinical trials or observational studies); this guidance will be used for interpreting the findings of our meta-analyes.

Overall, we aim to develop evidence updates on the maintenance treatment of asthma and management of acute severe asthma attacks that will cover all available evidence, carefully considering methodological limitations. These will be used 
by the PeARL Think Tank for the development of clinical recommendations and to guide future clinical research.

\section{Author affiliations}

${ }^{1}$ Division of Infection, Immunity and Respiratory Medicine, The University of

Manchester, Manchester, UK

${ }^{2}$ North West Lung Centre, Manchester University NHS Foundation Trust, Manchester, UK

${ }^{3}$ Athens Breath Centre, Athens, Greece

${ }^{4}$ First Department of Pediatrics, "Aghia Sofia" Children's Hospital, University of Athens, Athens, Attica, Greece

${ }^{5}$ Department of Cardiovascular and Thoracic Sciences, Catholic University of the Sacred Heart, Milano, Lombardia, Italy

${ }^{6}$ Department of Biomedical Sciences, University of West Attica, Egaleo, Attica, Greece

${ }^{7}$ Department of Paediatrics, Imperial College London, London, UK

${ }^{8}$ Unité de Pneumologie et Allergologie Pédiatriques, Hôpital Jeanne de Flandre, CHU Lille, Lille, Hauts-de-France, France

${ }^{9}$ Pediatrics, University of Montreal, Montreal, Quebec, Canada

${ }^{10}$ Pediatric Allergy and Asthma Unit, Hacettepe Universitesi, Ankara, Turkey

${ }^{11}$ Pulmonology and Allergy Unity, La Fe University and Polytechnic Hospital, Valencia,

Comunidad Valenciana, Spain

${ }^{12}$ Pediatric Allergy and Immunology, Children's Hospital Boston, Boston,

Massachusetts, USA

${ }^{13}$ Centre of Academic Primary Care, University of Aberdeen, Aberdeen, UK

${ }^{14}$ Observational and Pragmatic Research Institute, Singapore

${ }^{15}$ Asthma UK Centre for Applied Research, Usher Institute of Population Health

Sciences and Informatics, The University of Edinburgh, Edinburgh, UK

${ }^{16}$ Allergy and Clinical Immunology, Transylvania University, Brasov, Romania

${ }^{17}$ Department of Allergy, Immunology, and Pulmonary Medicine, University of Washington, Seattle, Washington, USA

${ }^{18}$ Institute of Infection and Global Health, University of Liverpool, Liverpool, UK

${ }^{19}$ Acute Intensive Care Unit, University Hospital of South Manchester NHS

Foundation Trust, Manchester, UK

${ }^{20}$ National Heart and Lung Institute (NHLI), Imperial College London, London, UK

${ }^{21}$ Department of Pediatrics, Pontifical Universidad Catolica de Chile, Santiago, Chile

${ }^{22}$ Allergy and Airway Diseases Patient's Associations, European Federation of

Pharmaceutical Industries and Associations, Brussels, Belgium

${ }^{23}$ Allergy, Asthma and Immunology, Penn State University, Hershey, Pennsylvania, USA

${ }^{24}$ Department of Respiratory Medicine and Allergology, Institute for Clinical Science,

Skane University Hospital Lund Hematological Clinic, Lund, Skåne, Sweden

${ }^{25}$ Department of Respiratory Medicine, First Faculty of Medicine, Charles University and Thomayer Hospital, Prague, Czech Republic

${ }^{26}$ Department of Clinical Pharmacy \& Pharmacology, University of Groningen, University Medical Center of Groningen and QPS-NL, Groningen, Netherlands

${ }^{27}$ Department of Pediatric Pulmonology and Allergy, Medical University of Warsaw, Warszawa, Poland

${ }^{28}$ Department of Pediatrics and Medicine, University of Wisconsin School of

Medicine and Public Health, Madison, Wisconsin, USA

${ }^{29}$ Centre for Genomics and Child Health, Blizard Institute, Queen Mary University of London, London, UK

${ }^{30}$ Department of Women's and Children's Health, Karolinska Institute, Stockholm, Stockholm, Sweden

${ }^{31}$ Pediatric Allergy and Immunology Unit, Ain Shams University, Cairo, Egypt

${ }^{32}$ Department of Social Medicine, Faculty of Medicine, University of Crete, Rethimno, Greece

${ }^{33}$ Department of Paediatrics, University of Turku, Turku, Finland

${ }^{34}$ Family Physician, Airways Group of Canada, University of Toronto, Toronto, Ontario, Canada

${ }^{35}$ School of Paediatrics and Child Health, University of Western Australia, Perth, Western Australia, Australia

${ }^{36}$ Department of Allergy, University of Helsinki, Helsinki, Uusimaa, Finland

${ }^{37}$ Department of Pediatric Pulmonology, Immunology and Intensive Care Medicine,

Charité - University Medicine, Berlin, Germany

${ }^{38}$ Third Department of Paediatrics, National and Kapodistrian University of Athens School of Health Sciences, Athens, Greece

${ }^{39}$ Allergy Center, Hospital CUF Descobertas, Lisboa, Portugal

${ }^{40}$ Allergy \& Asthma, VN, Chennai, India
${ }^{41}$ Allergy Center, CUF Infante Santo Hospital, Lisbon, Portugal

${ }^{42}$ Chronic Diseases Research Center (CEDOC), NOVA Medical School / Faculdade de Ciências Médicas, Universidade NOVA de Lisboa, Lisbon, Portugal

${ }^{43}$ Laboratory of Respiratory Physiology, Infant Center, School of Medicine, Pontifícia Universidade Católica do Rio Grande do Sul (PUCRS), Porto Alegre, Brazil

${ }^{44}$ Paediatric Department, Motol University Hospital, Praha, Czech Republic

${ }^{45}$ The David Hide Asthma and Allergy Research Centre, St Mary's Hospital, Newport Isle of Wight, UK

${ }^{46}$ Faculty of Medicine, Clinical and Experimental Sciences and Human Development in Health Academic Units, University of Southampton, Southampton, UK

${ }^{47} \mathrm{NIHR}$ Biomedical Research Centre, University Hospital Southampton NHS

Foundation Trust, Southampton, UK

${ }^{48}$ Department of Child Health, University of Aberdeen, Aberdeen, UK

${ }^{49}$ Department of Respiratory Medicine and Department of Pediatric Pulmonology,

University of Amsterdam, Amsterdam, Netherlands

${ }^{50}$ Allergy \& Asthma, Global Patient Platform, Virginia, Virginia, USA

${ }^{51}$ Department of Paediatrics, Faculty of Medicine, The Chinese University of Hong Kong, Sha Tin, Hong Kong

${ }^{52}$ Allergy Department, 2nd Paediatric Clinic, National and Kapodistrian University of Athens, Athens, Attica, Greece

${ }^{53}$ Department of Paediatrics and Child Health, Red Cross War Memorial Children's Hospital, University of Cape Town, Rondebosch, Western Cape, South Africa

${ }^{54}$ Unit on Child and Adolescent Health, Medical Reaserch Council, Cape Town, South Africa

Twitter Alexander G Mathioudakis @mathioudakisag

Acknowledgements AGM was supported by the National Institute of Health Research Manchester Biomedical Research Centre (NIHR Manchester BRC). We thank Mrs Maria Kritikou for excellent administrative support of the study

Contributors Study conception: AGM and NGP. Study design: AGM, MM and NGP Preparation of the manuscript: AGM. Critical revision and final approval of the manuscript: AGM, MM, CB, GSA, AC, AD, FMD, OK, CM, ANG, WP, DP, AS, IOA, LB, AB AB, MB, JAC-R, GDC, TC, ZD, WF, TF, JEG, JG, GH, EMH, DI, TJ, AK, RFL, PNLS, MJM, GAM, PM, MM, MM-A, KN, EP, HP, PMCP, PP, GR, IT, ST, VS, TAW, GWKW, PX, HJZ and NGP.

Funding This work was supported by the Respiratory Effectiveness Group (REG). REG has received support from AstraZeneca, Novartis and Sanofi for continued work on PeARL. (Award/Grant name: PeARL, Award/Grant Number: N/A). This is an investigator initiated study and the funders were not involved in the selection of the topic, or design of these systematic reviews. AGM was supported by the National Institute for Health Research Manchester Biomedical Research Centre (NIHR Manchester BRC).

Competing interests AGM reports grants from Boehringer Ingelheim outside the submitted work. AC reports personal fees from Novartis, Regeneron / Sanofi, Thermo Fisher Scientific, Boehringer Ingelheim and Philips, outside the submitted work. LB reports personal fees from Aerocrine, GlaxoSmithKline, Genentech/ Novartis, Merck, DBV Technologies, Teva, Boehringer Ingelheim, AstraZeneca, WebMD/Medscape, Sanofi/Regeneron, Vectura and Circassia outside the submitted work. TC reports grants and personal fees CSL Behring, Dyax, Takeda, BioCryst, Pharming, personal fees from Grifols, grants and non-financial support from GSK, Regeneron, Novartis/Genetech outside the submitted work. AD reports grants and personal fees from Stallergenes Greer, personal fees from Novartis, ALK, TEVA, GSK, MEDA-MYLAN, CHIESI, Almmune, DBV technologies and Astra Zeneca, outside the submitted work. ZD reports personal fees from academic affiliations, ZD acts as Executive and Scientific Medical Director at a phase I/II pharmacological unit (QPS-NL), which performs clinical studies for pharmaceutical companies. ZD reports personal fees from Astrazeneca, ALK, Aquilon, Boehringer Ingelheim, CSL, HAL Allergy, MSD, and Sanofi-Genzyme outside the submitted work. FMD reports grants from Thorasys; personal fees from Jean-Coutu Pharmaceuticals, unrestricted research funds from Novartis Canada, Teva and Trudell Medical, research grants from GlaxoSmithKline and MEDteq in partnership with Thorasys; honorarium for consultancy work from Covis Pharma and Teva; and honorarium as invited speaker from Covis Pharma, Pharmacy Brunet, outside the submitted work. JEG reports grants from NIH/NIAID, personal fees from Regeneron, Ena Theraputics and Medlmmune outside the submitted work; personal fees and stock options from Meissa Vaccines Inc outside the submitted work. JG reports personal fees from GSK, Vifor Pharmaceuticals, Novartis, BV Pharma and AstraZeneca outside the submitted work. AK reports personal fees Astra Zeneca, Behring, Boehringer Ingelheim, Covis, GSK, NovoNordisk, Novartis, Griffols, Pfizer, 
Sanofi, Teva and Trudel, outside the submitted work. RFL reports grants from NIH, non-financial support from GlaxoSmithKline, Boehringer-Ingelheim, Merck, TEVA, American Academy of Allergy, Asthma and Immunology, grants from Clinical and Translational Science Award (NIH), Childhood Origins of ASThma (COAST) grant, AsthmaNet, personal fees from LSU, Elsevier, UpToDate, the University of Kentucky, ThermoFischer, and Food Allergy Research and Education (FARE) Network, outside the submitted work. CM reports personal fees from Novartis, GSK, Astra Zeneca, Thermo Fisher and Boehringer Ingelheim outside the submitted work. NGP reports personal fees from ALK, Novartis, Nutricia, HAL, Menarini/FAES Farma, Sanofi, Mylan/MEDA, Biomay, AstraZeneca, GSK, MSD, ASIT BIOTECH and Boehringer Ingelheim; grants from Gerolymatos International SA and Capricare outside the submitted work. WP reports grants from NIH; grants and personal fees from Genentech/Novartis, Sanofi/Rgeneron; personal fees GSK; non-financial support from Thermo Fisher, Lincoln Diagnostics, Alk Abello, and Monaghen, outside the submitted work. PP reports grants from Astra Zeneca, Chiesi and TEVA; personal fees from Astra Zeneca, TEVA, Novartis, Mundipharma, S\&D Pharma, and GlaxoSmithKline outside the submitted work. DP reports grants from AKL Research and Development, British Lung Foundation, Respiratory Effectiveness Group and the UK National Health Service; grants and personal fees from Boehringer Ingelheim, Chiesi, Circassia, Mylan, Mundipharma, Napp, Novartis, Pfizer, Regeneron Pharmaceuticals, Sanofi Genzyme, TEVA, Theravance and Zentiva (Sanofi Generics); personal fees from Cipla, GlaxoSmithKline, Kyorin and Merck; non-financial support from Efficacy and Mechanism Evaluation programme, Health Technology Assessment, outside the submitted work; DP also reports stock/stock options from AKL Research and Development which produces phytopharmaceuticals; and owns $74 \%$ of the social enterprise Optimum Patient Care (Australia and UK) and $74 \%$ of Observational and Pragmatic Research Institute (Singapore), outside the submitted work. GR reports personal fees from ALK, Allergen Therapeutics, Meda Plus, Merck and a patent for the use of sublingual immunotherapy to prevent the development of allergy in at-risk infants, outside the submitted work. IT reports personal fees from Novartis, GSK, Boehringer Ingelheim and Astra Zeneca; grants from GSK Hellas, outside the submitted work. PX reports personal fees from Nutricia, Nestle, Friesland, Uriach, Novartis Pharma AG, and GlaxoSmithkline outside the submitted work.

\section{Patient consent for publication Not required.}

Provenance and peer review Not commissioned; externally peer reviewed.

Supplemental material This content has been supplied by the author(s). It has not been vetted by BMJ Publishing Group Limited (BMJ) and may not have been peer-reviewed. Any opinions or recommendations discussed are solely those of the author(s) and are not endorsed by BMJ. BMJ disclaims all liability and responsibility arising from any reliance placed on the content. Where the content includes any translated material, BMJ does not warrant the accuracy and reliability of the translations (including but not limited to local regulations, clinical guidelines, terminology, drug names and drug dosages), and is not responsible for any error and/or omissions arising from translation and adaptation or otherwise.

Open access This is an open access article distributed in accordance with the Creative Commons Attribution Non Commercial (CC BY-NC 4.0) license, which permits others to distribute, remix, adapt, build upon this work non-commercially, and license their derivative works on different terms, provided the original work is properly cited, appropriate credit is given, any changes made indicated, and the use is non-commercial. See: http://creativecommons.org/licenses/by-nc/4.0/.

\section{ORCID iDs}

Alexander G Mathioudakis http://orcid.org/0000-0002-4675-9616

Apostolos Beloukas http://orcid.org/0000-0001-5639-0528

Gary WK Wong http://orcid.org/0000-0001-5939-812X

Nikolaos G Papadopoulos http://orcid.org/0000-0002-4448-3468

\section{REFERENCES}

1 To T, Stanojevic S, Moores G, et al. Global asthma prevalence in adults: findings from the cross-sectional World health survey. BMC Public Health 2012:12:204.

2 Global Initiative for Asthma. GINA guidelines. global strategy for asthma management and prevention. Available: www.ginasthma.org [Accessed 1 Mar 2018].

3 British Thoracic Society, Scottish Intercollegiate Guidelines Network. Sign 158: British guideline on the management of asthma. Edinburgh: BTS/SIGN, 2019.

4 Papadopoulos NG, Čustović A, Cabana MD, et al. Pediatric asthma: an unmet need for more effective, focused treatments. Pediatr Allergy Immunol 2019;30:7-16.
5 Papadopoulos NG, Arakawa H, Carlsen K-H, et al. International consensus on (ICON) pediatric asthma. Allergy 2012;67:976-97.

6 Kwong CG, Bacharier LB. Phenotypes of wheezing and asthma in preschool children. Curr Opin Allergy Clin Immunol 2019;19:148-53.

7 Tse SM, Krajinovic M, Chauhan BF, et al. Genetic determinants of acute asthma therapy response in children with moderate-to-severe asthma exacerbations. Pediatr Pulmonol 2019;54:378-85.

8 EMA. Multidisciplinary scientific guidelines: paediatrics, 2013. Available: http://www.ema.europa.eu/ema/index.jsp?curl= pages/regulation/general/general_content_000404.jsp\&mid= WC0b01ac0580029572

9 Turner MA, Catapano M, Hirschfeld S. Global research in paediatrics. paediatric drug development: the impact of evolving regulations. Adv Drug Deliv Rev 2014;73:2-13.

10 Mathioudakis AG, Custovic A, Deschildre A, et al. Research priorities in pediatric asthma: results of a global survey of multiple Stakeholder groups by the pediatric asthma in real life (pearl) think tank. $J$ Allergy Clin Immunol Pract 2020;8:1953-60.

11 Mathioudakis AG, Miligkos M, Papadopoulos NG. Maintenance treatment for childhood asthma: a protocol for an overview of systematic reviews by the pearl think-tank. International prospective register of systematic reviews, 2020.

12 Mathioudakis AG, Alimani GS, Papadopoulos NG. Pharmacological management of acute severe asthma attacks in children. protocol for a systematic review by the pearl think tank. International prospective register of systematic reviews, 2020.

13 Higgins JPT. Cochrane Handbook for systematic reviews of interventions. The Cochrane Collaboration, 2011.

14 Moher D, Liberati A, Tetzlaff J, et al. Preferred reporting items for systematic reviews and meta-analyses: the PRISMA statement. $J$ Clin Epidemiol 2009;62:1006-12.

15 Thomson D, Russell K, Becker L, et al. The evolution of a new publication type: steps and challenges of producing overviews of reviews. Res Synth Methods 2010;1:198-211.

16 Craig SS, Dalziel SR, Powell CV, et al. Interventions for escalation of therapy for acute exacerbations of asthma in children: an overview of Cochrane reviews. Cochrane Database Syst Rev 2020;8:CD012977.

17 Katsunuma T, Fujisawa T, Maekawa T, et al. Low-Dose l-isoproterenol versus salbutamol in hospitalized pediatric patients with severe acute exacerbation of asthma: a double-blind, randomized controlled trial. Allergol Int 2019;68:335-41.

18 Alshehri M, Almegamesi T, Alfrayh A. Efficacy of nebulized furosemide in children with moderate attack of asthma. West Afr J Med 2005;24:246-51.

19 Douglas LC, Choi J, Esteban-Cruciani N. Azithromycin treatment in children hospitalized with asthma: a retrospective cohort study. $J$ Asthma 2020;57:525-31.

20 Allen JY, Macias CG. The efficacy of ketamine in pediatric emergency department patients who present with acute severe asthma. Ann Emerg Med 2005;46:43-50.

21 Mathioudakis A, Chatzimavridou-Grigoriadou V, Evangelopoulou E, et al. Vasoactive intestinal peptide inhaled agonists: potential role in respiratory therapeutics. Hippokratia 2013;17:12-16.

22 Robroeks CMHHT, van de Kant KDG, van Vliet D, et al. Comparison of the anti-inflammatory effects of extra-fine hydrofluoroalkanebeclomethasone vs fluticasone dry powder inhaler on exhaled inflammatory markers in childhood asthma. Ann Allergy Asthma Immunol 2008;100:601-7.

23 Tantichaiyakul P, Preutthipan A. Ketotifen versus inhaled budesonide for controlling childhood asthma. J Med Assoc Thai 2010;93:541-9.

24 Shea BJ, Reeves BC, Wells G, et al. AMSTAR 2: a critical appraisal tool for systematic reviews that include randomised or nonrandomised studies of healthcare interventions, or both. $B M J$ 2017;358:j4008.

25 Pollock M, Fernandes RM, Hartling L. Evaluation of AMSTAR to assess the methodological quality of systematic reviews in overviews of reviews of healthcare interventions. BMC Med Res Methodol $2017 b ; 17: 13$.

26 Sterne JAC, Savović J, Page MJ, et al. Rob 2: a revised tool for assessing risk of bias in randomised trials. BMJ 2019;366:14898.

27 Campbell JD, Perry R, Papadopoulos NG, et al. The real life evidence assessment tool (relevant): development of a novel quality assurance asset to rate observational comparative effectiveness research studies. Clin Transl Allergy 2019;9:21.

28 Balshem H, Helfand M, Schünemann HJ, et al. Grade guidelines: 3. Rating the quality of evidence. J Clin Epidemiol 2011;64:401-6.

29 Guyatt GH, Ebrahim S, Alonso-Coello P, et al. Grade guidelines 17: assessing the risk of bias associated with missing participant outcome data in a body of evidence. J Clin Epidemiol 2017;87:14-22 
30 Papadopoulos NG, Custovic A, Deschildre A, et al. Impact of COVID-19 on pediatric asthma: practice adjustments and disease burden. J Allergy Clin Immunol Pract 2020;8:2592-9.

31 Papadopoulos NG, Mathioudakis AG, Custovic A, et al. Childhood asthma outcomes during the COVID-19 pandemic: findings from the pearl multi-national cohort. Allergy 2021;76:1765-75.

32 Vézina K, Chauhan BF, Ducharme FM. Inhaled anticholinergics and short-acting beta(2)-agonists versus short-acting beta2-agonists alone for children with acute asthma in hospital. Cochrane Database Syst Rev 2014;7:CD010283.
33 Normansell R, Kew KM, Mathioudakis AG. Interventions to improve inhaler technique for people with asthma. Cochrane Database Syst Rev 2017;3:CD012286.

34 Normansell R, Kew K, Stovold E, et al. Interventions to improve inhaler technique and adherence to inhaled corticosteroids in children with asthma. Paediatr Respir Rev 2017;23:53-5.

35 Knightly R, Milan SJ, Hughes R, et al. Inhaled magnesium sulfate in the treatment of acute asthma. Cochrane Database Syst Rev 2017;11:CD003898. 